UCRL-JC-125849

PREPRINT

\title{
Radiation MHD Modeling of a Proposed Dynamic Hohlraum
}

\author{
J. H. Hammer, J. S. De Groot, M. Tabak, \\ A. Toor, G. B. Zimmerman
}

This paper was prepared for submittal to Fourth International Conference on Dense Z-Pinch Vancouver, Canada

May 28-30, 1997

July 15, 1997

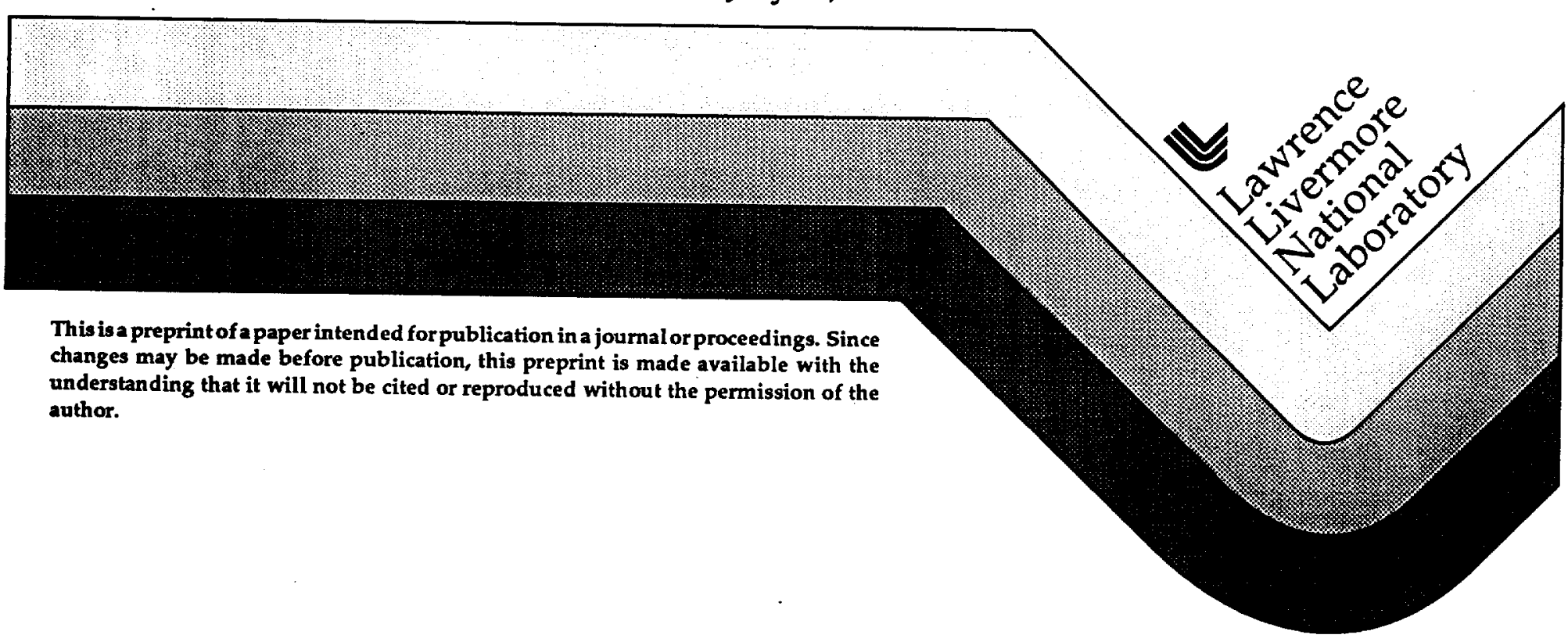




\section{DISCLAIMER}

This document was prepared as an account of work sponsored by an agency of the United States Government. Neither the United States Government nor the University of California nor any of their employes, makes any warranty, express or implied, or assumes any legal liability or responsibility for the accuracy, completeness, or usefulness of any information, apparatus, product, or process disclosed, or represents that its use would not infringe privately owned rights. Reference herein to any specific commercial product, process, or service by trade name, trademark, manufacturer, or otherwise, does not necessarily constitute or imply its endorsement, recommendation, or favoring by the United States Government or the University of California. The views and opinions of authors expressed herein do not necessarily state or reflect those of the United States Government or the University of California, and shall not be used for advertising or product endorsement purposes. 


\title{
Radiation MHD Modeling of a Proposed Dynamic Hohlraum
}

\author{
James H. Hammer ${ }^{1}$, John S. De Groot ${ }^{1,2}$, Max Tabak ${ }^{1}$, Art Toor ${ }^{1}$ \\ and George B. Zimmerman \\ 'Lawrence Livermore National Laboratory, Livermore California 94550 \\ ${ }^{2}$ Plasma Research Group and Department of Applied Science, University of California, Davis, CA \\ 95616
}

In this paper we report 2D radiation magnetohydrodynamic simulations of a dynamic hohlraum target designed to be driven by the $Z$ accelerator (1) at Sandia National Laboratory, Albuquerque New Mexico. $Z$ generates currents up $20 \mathrm{MA}$ with a rise time of $100 \mathrm{~ns}$ and peak electrical power of $40 \mathrm{TW}$. In this design we attempt to reduce the effects of magneto-Rayleigh Taylor (RT) modes by using a distributed initial density profile. Earlier work $(2,3)$ showed that "tailoring" the initial density profile could reduce the sheath acceleration and the number of e-foldings that the RT instability grows during the implosion. As the sheath moves in radially, fresh material is swept up or "snow plowed", providing a back pressure that counters the $\mathrm{J} \times \mathrm{B}$ force. A special profile can be found in which the unstable outer surface of the sheath implodes at constant velocity, reducing the classical growth rate to zero, although residual Richtmeyer-Meshkov type instability (instability of the snow-plow shock front) may be present. In practice, it is hard to create tailored initial density profiles due to the difficulty of machining and otherwise manipulating very low density materials. It becomes easier to manufacture these complex targets as the current, energy and load mass increase with large drivers. $Z$ is the first fast pulse power device with enough energy to consider loads of this type. 


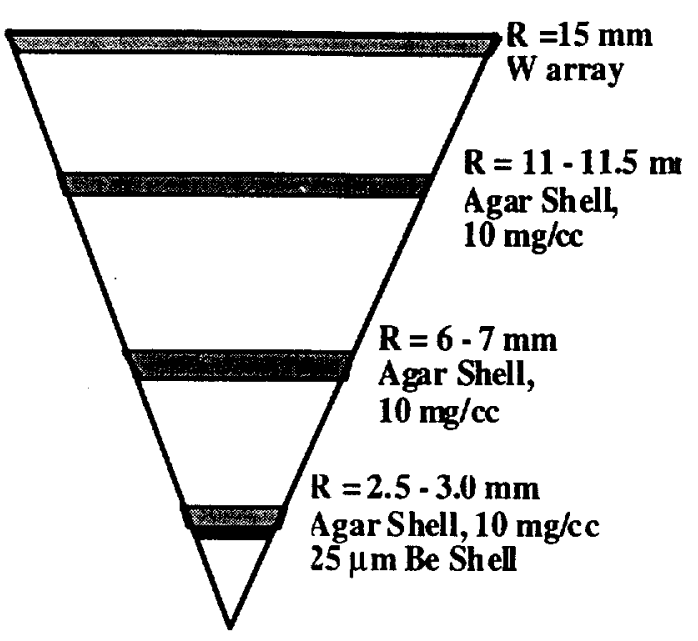

Fig. 1 Dynamic hohlraum target
The design we have modeled employs a $3 \mathrm{~cm}$ diameter, $2 \mathrm{~cm}$ long, tungsten wire array of mass $6 \mathrm{mg} / \mathrm{cm}$ as the high atomic number shell material. The wire array implodes on 3 concentric, annular shells of low density $(10 \mathrm{mg} / \mathrm{cc})$ agar foam with a $25 \mathrm{~mm}$ layer of beryllium covering the interior of the central shell, shown in Fig. 1. The annular shells, with foam between radii of 1.1 and $1.15 \mathrm{~cm}$ (outer shell), 0.6 and $0.7 \mathrm{~cm}$ (middle shell) and 0.25 and $0.3 \mathrm{~cm}$ (inner shell), explode under the influence of the X-rays emitted by the wire array and outer shells. In

the process, they create a rough approximation to the zero-acceleration tailored density profile. The use of multiple shells to control RT instability in Z-pinches has been studied previously (4). 1D simulations show the stagnation of the

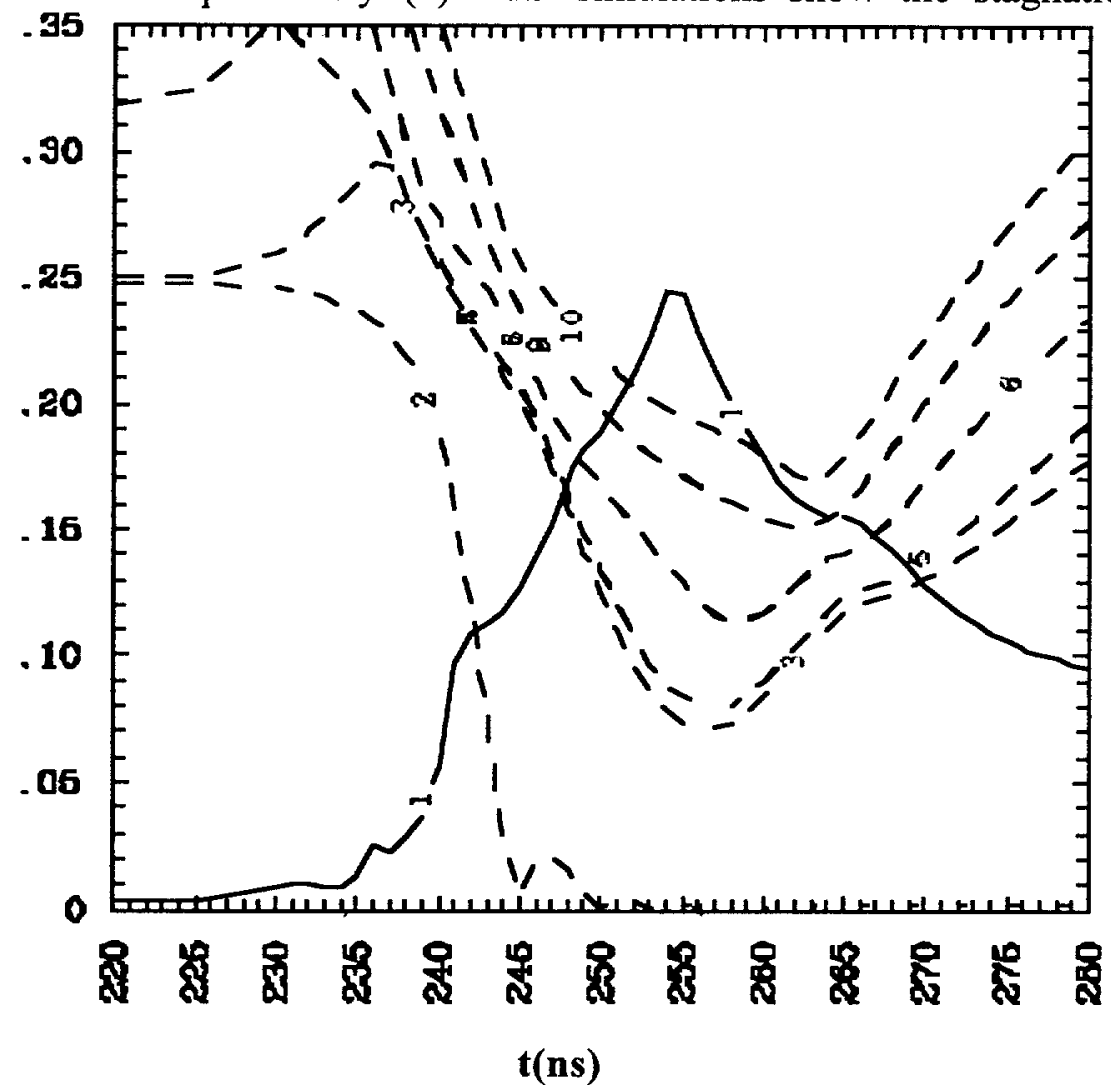

Figure 2. $\operatorname{Tr}(\mathrm{keV})$ on axis and $\mathbf{r}(\mathrm{cm})$ of shells vs. time 
beryllium at a minimum diameter of about $0.16 \mathrm{~cm}$, with a peak temperature of $240 \mathrm{eV}$ and temperatures exceeding $200 \mathrm{eV}$ for $7 \mathrm{~ns}$. The radii of the different layers and the radiation temperature on the pinch axis are shown in Fig. (2). The peak temperature occurs $50 \mathrm{~ns}$ after the current peaks at $17 \mathrm{MA}$. In the hot, stagnated beryllium the average distance X-ray photons can travel before being absorbed and re-emitted, the Rosseland mean free path, is several times the pinch diameter, while the agar and tungsten layers have an optical depth of about 20. With these conditions, it is sensible to describe the configuration as a dynamic hohlraum. The beryllium fills the hohlraum at a density of about $0.1 \mathrm{~g} / \mathrm{cc}$ and pressure of order $50 \mathrm{Mbar}$. This type of hohlraum is better suited to driving auxiliary experiments than ICF capsules inside the hohlraum that could be affected by the high plasma pressure. The drive temperature seen by an auxiliary load depends on the type of experiment. A highly reflective load on the end sees the full $240 \mathrm{eV}$, while a highly absorptive load removes significant energy from the hohlraum, dropping the effective drive temperature to $180 \mathrm{eV}$.

We've also done 2D calculations including the effects of RT modes for this target and similar designs. A calculation for a similar target with a $1 \%$ density perturbation and a $2 \mathrm{~mm}$ wavelength shows significant instability growth in the tungsten, but little perturbation of the inner layers. At stagnation against the inner annulus, the bubble-spike formation in the tungsten is partly reversed. The peak temperature drops from $230 \mathrm{eV}$ to $\sim 220 \mathrm{eV}$, and the hohlraum interior remains optically thin at about the same diameter as the 1D simulation. 2D periodic simulations with $5 \%$ initial random zone-to-zone density perturbation show larger amplitude growth in the tungsten with small perturbation of the inner shells. The tungsten is sufficiently disrupted that cracks in the radiation case form and allow large radial losses, causing the radiation temperature on axis to drop to $180 \mathrm{eV}$. Perturbations in the $1-5 \%$ range have been found to give reasonable pulsewidths for Saturn and $Z$ experiments $(2,6)$, although the perturbation level imposed in this way is zoning dependent.

To reduce the uncertainty in choosing the perturbation level, and come closer to a "first principles" RT calculation, we have done simulations with a very fine mesh. As discussed in earlier work (5), RT modes at short wavelengths can grow to nonlinear amplitudes very quickly from small initial perturbations, then seed more destructive long wavelength modes through a nonlinear cascade. To provide a self-consistent instability seed, we have done a finely zoned (10 micron $x 10$ micron zones) calculation capable of resolving the short wavelengths that dominate the early phase. This method of initiating RT calculations with a $2 \mathrm{D} \mathrm{r}-\mathrm{z}$ code is only valid when there are enough wires in the initial array to merge into an azimuthally uniform sheath before significant RT growth begins. For this calculation, modeling $3 \mathrm{~mm}$ of the pinch length with periodic boundary conditions in the z-direction, random density perturbations of $0.1 \%$ were sufficient to 


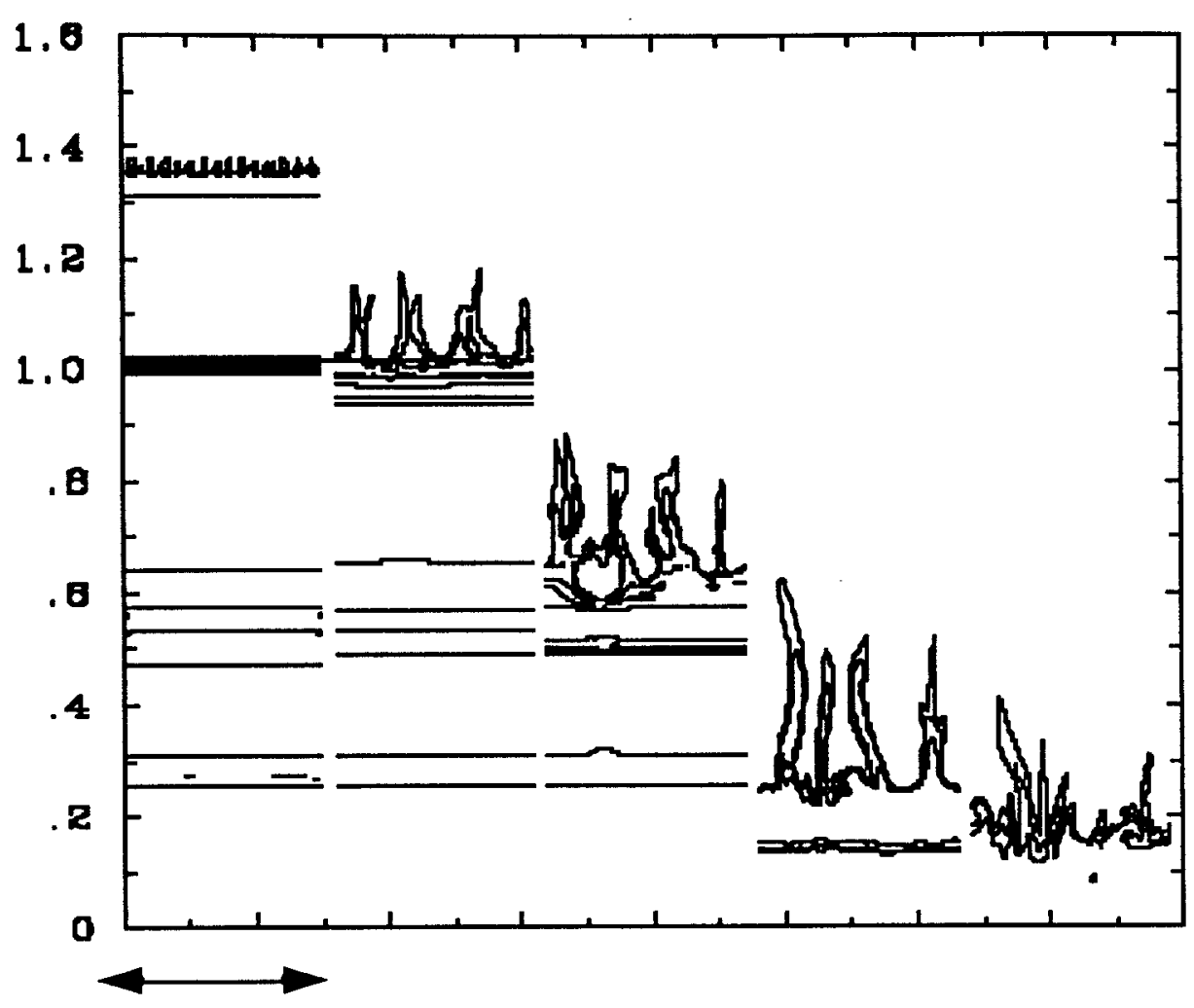

$0.3 \mathrm{~cm}$ axial extent

Figure 3. Density contours at $20 \mathrm{~ns}$ intervals

stimulate large amplitude RT growth. Once the dominant features were several hundred microns in scale, we linked to a coarser mesh ( $\sim 30$ micron zone size) to follow the complete implosion. A series of time snapshots of the plasma density for the multi-shell agar foam + beryllium target are shown in Fig. (3). This calculation showed reasonable integrity of the radiation case so that radiation leakage was not a major effect and the radiation temperature was $\sim 220 \mathrm{eV}$ in the hohlraum interior. A plume of optically-thick high $Z$ material reached the axis at one location, however, which could inhibit energy flow to an adjacent load. If the high-Z plume occurs more than a few $\mathrm{mm}$ from the output end, the effect on energy flow should be small. The RT calculations do not include the effect of an $\mathrm{X}$-ray-absorbing load, which would cause an additional drop in the radiation temperature.

All of the calculations show significant breakup of the tungsten sheath before it encounters the outermost stabilizing agar shell. This prompts us to consider an initial state that more closely approximates the zero-acceleration density profile at large radius. At present, it is not practical to extend the profile to larger radius with low density foams because of the limited ability to machine and handle these extremely fragile materials. An alternative may be to use 
concentric, nested wire arrays, with the separation between wires in each shell smaller than the distance the wires expand due to heating by $\mathrm{X}$-rays. With this technique, an arbitrary initial density profile of virtually any material may be possible. The first experiments with simpler loads will tell us if these more complex targets are necessary to control RT instability.

\section{Acknowledgments}

Work Performed under the auspices of the U. S. Department of Energy by the Lawrence Livermore National Laboratory under Contract W-7405-ENG-48 and Sandia National Laboratories under Contract DE-AC04-94AL85000.

\section{References}

1. R.B. Spielman, et. al., Paper 0-4-3 in Proceedings of the 10th International Conference on High Power Particle Beams, Prague, Czech Republic, June 10-14, 1996, ed. K. Jungwirth and J. Ullschmied, pp. 150-153. Copies of the conference proceedings can be ordered from BEAMs96, Institute of Plasma Physics, Czech Academy of Sciences, Za Slovankou 3, 18200 Prague, Czech Republic.

R.B. Spielman, G. A. Chandler, C. Deeney, F. Long, T.H. Martin, M.K. Matzen, D.H. McDaniel, T.J. Nash, J.L. Porter, L.E. Ruggles, T.W.L. Sanford, J.F. Seamen, W.A. Stygar, S.P. Breeze, J.S. McGurn, J.A. Torres, D.M. Zagar, T.L. Gilliland, D. Jobe, K.W. Struve, M. Mostrom, P. Corcoran, I. Smith, R.W. Shoup, "PBFA Z: A 20-MA Driver for Z-Pinch Radiation Sources", Bull. Am. Phys. Soc. 41, 1422 (1996).

2. J.H. Hammer, J.L. Eddleman, P.T. Springer, M. Tabak, A. Toor, K. Wong, G.B. Zimmerman, C. Deeney, R. Humphreys, T.J. Nash, T.W.L. Sanford, R.B. Spielman, J.S. De Groot, "Two-dimensional radiation-magnetohydrodynamic simulations of SATURN imploding $Z$ pinches", Phys. Plasmas, 3, 2063 (1996).

3. A.L. Velikovich, F.L. Cochran, J. Davis, "Suppression of Rayleigh-Taylor Instability in Zpinch Loads with Tailored Density Profiles", Phys. Rev. Lett., 77, 853 (1996).

4. R.B. Baksht, S.P. Bugaev, I.M. Datsko, A.V. Luchinskii, V.I. Oreshkin, A.V. Fedyunin, Yu.D. Korolev, I.A. Shemyakin, and V.G. Rabotkin "Dense Z-Pinches, Tjore International Conference", ed. by M. Haines and A. Knight (AIP Conference Proceedings, vol. 299, p. 365, AIP, New York), 1994.

5. J.H. Hammer, J.L. Eddleman, M. Tabak, A. Toor and G.B. Zimmerman, "Sheath Broadening in Imploding Z-pinches due to Large-bandwidth Rayleigh-Taylor Instability", in Proceedings of the 10th International Conference on High Power Particle Beams, Prague, Czech Republic, June 10-14, 1996, ed. K. Jungwirth and J. Ullschmied, pp. 150-153. Copies of the conference proceedings can be ordered from BEAMs96, Institute of Plasma Physics, Czech Academy of Sciences, Za Slovankou 3, 18200 Prague, Czech Republic.

6. D.L. Peterson, R.L. Bowers, J.H. Brownell, A.E. Greene, K.D. McLenithan, T.A. Oliphant, N.F. Roderick and A.J. Scannapieco, "Two-dimensional modeling of magnetically-driven Rayleigh-Taylor instabilities in cylindrical Z pinches", Phys. Plasmas, 3, 368. 


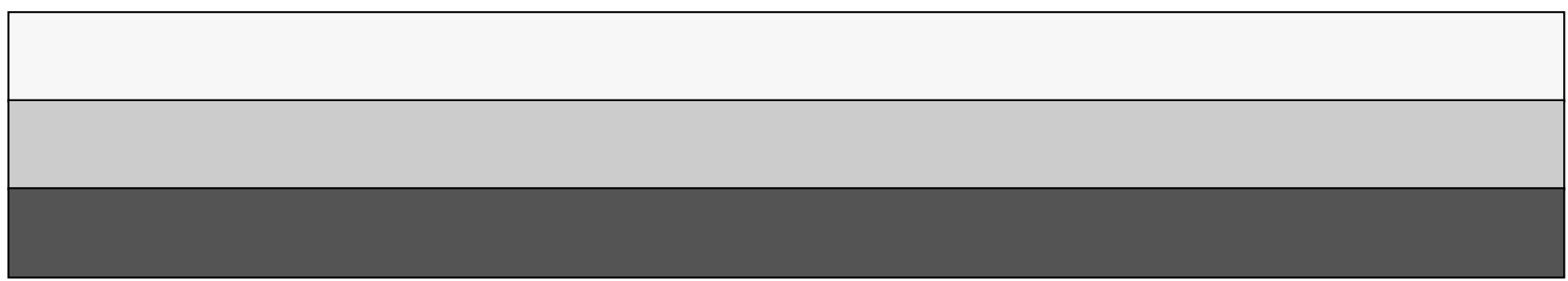

